\title{
Editorial on EMC 2014 special issue
}

\author{
Masataka Kawai · Graham Lamb · Stefan Galler
}

Published online: 2 December 2014

(C) Springer International Publishing Switzerland 2014

This Special Issue showcases one view point, one original work, and four review articles covering a range of important aspects of skeletal and cardiac muscle functions in health and disease. These topics include little known history of muscle biology in the nineteenth century, the role of $\beta$-tropomyosin in contraction, the role of titin in muscle elasticity and its modulation by calcium, oxidation and other stresses, the determinants of contractile functions in cardiomyocytes, the way in which their defects contribute to heart failure, and how defects in calcium storage and release underlie central core disease (CCD) and exertional heat stroke (EHS). The Special Issue also includes 270 contributed abstracts that were presented during the conference.

The first article is by Stefan Galler, the EMC chair for 2014. It provides a very interesting historic viewpoint on muscle biology. Dr. Galler reports that the fine structure of cross-striated muscle and its changes during contraction were somewhat known in the nineteenth century (Galler 2014). This knowledge resulted from excellent light microscopy carried out especially on muscle fibres with long sarcomeres (leg muscles of the water beetle Hydrophilus piceus). A major protagonist of this research was Ernst Brücke, professor of physiology at the University of

M. Kawai ( $\square)$

Department of Anatomy and Cell Biology, University of Iowa,

Iowa City, IA 52242, USA

e-mail:masataka-kawai@uiowa.edu

G. Lamb

School of Life Sciences, La Trobe University, Melbourne,

VIC 3086, Australia

e-mail: g.lamb@latrobe.edu.au

S. Galler

Department of Cell Biology, Faculty of Natural Sciences, University of Salzburg, 5020 Salzburg, Austria
Vienna, Austria. Brücke (1858) studied birefringence properties of muscle fibres and came to the conclusion that the A-band of the sarcomere was composed of anisotropic filamentous materials which ran in parallel to the fibre axis. Krause (1869) later found that the isotropic I-band shortened during activation, whereas the A-band did not. This knowledge was seemingly forgotten in the first half of the twentieth century before it was rediscovered in 1954, when the sliding filament theory was formulated. Dr. Galler suggests that this loss of knowledge was the result of premature assumptions among the scientific community, and he concludes that, in doing science, there may be a danger in following the fashion of the time.

The original article by Scellini et al. in Poggesi-Tesi group is an interesting work examining the role of $\beta \beta \mathrm{Tm}$ (tropomyosin) in contractile activation and relaxation in single myofibrils from rabbit psoas muscle, with supporting data from in vitro motility assays (Scellini et al. 2014). Myofibrils are tiny, with a diameter of $1-3 \mu \mathrm{m}$ and the length $50-70 \mu \mathrm{m}$, and hence diffusion problems are virtually absent, but the preparation is difficult to handle because of its fragility and small size. For such a delicate task, the experimenter needs state of art equipment and well trained/controlled hands, which the Poggesi-Tesi group possesses. These investigators used Escherichia coli generated $\alpha \alpha \mathrm{Tm}$ and $\beta \beta \mathrm{Tm}$ and replaced native $\mathrm{Tm}$ in myofibrils. They observed, assuming a perfect replacement, that the rate of activation $\left(k_{\mathrm{ACT}}\right)$ and force redevelopment $\left(k_{\mathrm{TR}}\right)$ diminished when $\beta \beta \mathrm{Tm}$ was used instead of $\alpha \alpha \mathrm{Tm}$. They further observed that, with $\beta \beta \mathrm{Tm}$, isometric tension was not completely turned off at $\mathrm{pCa} 9$, indicating imperfect inhibition of the actin-myosin interaction. This was not the case with $\alpha \alpha \mathrm{Tm}$ at $\mathrm{pCa} 9$. The shorter slack sarcomere length observed with $\beta \beta T \mathrm{~m}$ in the relaxing condition relative to that with $\alpha \alpha \mathrm{Tm}$ was presumably 
related to the low level cross-bridge activation with $\beta \beta T \mathrm{Tm}$ in the relaxing solution. $\beta \beta \mathrm{Tm}$ exhibited slower relaxation time than $\alpha \alpha \mathrm{Tm}$ in the fast component of relaxation. The parameters which did not differ between $\alpha \alpha \mathrm{Tm}$ and $\beta \beta \mathrm{Tm}$ were: active tension, slow $k_{\mathrm{REL}}$, and cooperativity. There also may have been an effect on $\mathrm{Ca}^{2+}$ sensitivity, though the difference was not significant as measured by the in vitro motility assays. These findings provide new insight into the impact of tropomyosin isoform composition on thin filament regulation and force development.

The review by Beckendorf and Linke starts by providing an overview of the modifications of the thick and thin filaments in skeletal and cardiac muscle cells induced by oxidative stress, and then describes how oxidative stress modifies muscle elasticity and passive stiffness by acting on the giant elastic protein, titin (Beckendorf and Linke 2014). The authors describe four different mechanisms by which oxidative stress-related modifications can affect titin elasticity. The first two involve hypo-phosphorylation or oxidation of the $\mathrm{N} 2$-Bus region, a region present only in cardiac titin, with both effects being reversible and leading to increased titin stiffness. The other two mechanisms likely operate in all titin isoforms and involve $S$-glutathionylation of 'cryptic' cysteine residues in the immunoglobulin-like (Ig-) domains of titin which are exposed by stretch, and proteolytic cleavage at specific locations in the Ig-domains or linkers; both these mechanisms lead to a decrease in titin stiffness, but only the former is reversible. The authors conclude by discussing the possible net consequences of having such opposing, tissue-specific mechanisms for modulating titin stiffness, and also raise the possibility of using titin modifications as biomarkers of specific forms of cardiac and skeletal muscle disease, and of pharmacological targeting of titin as a therapeutic treatment.

The titin theme also carries into the review article by Rassier and colleagues. This article provides a useful description and historical overview of the phenomenon of 'static tension', in which skeletal muscle activation results in a small persistent increase in tension that is not attributable to actin-myosin interaction (Rassier et al. 2014). It was shown more than 30 years ago by independent studies that electrically-stimulated muscle fibres displayed an increase in fibre stiffness before the development of active force (Ford et al. 1981; Cecchi et al. 1982). Many researchers have studied this and other possibly related phenomena, and a number of theories have been advanced about the underlying mechanism(s). This review summarizes the data from a wide range of experiments with whole muscles to single fibres, and makes a case that the phenomenon is due in large part to a calcium-dependent increase in titin stiffness. The authors further discuss the important physiological consequences of having such a
$\mathrm{Ca}^{2+}$-induced increase in titin stiffness, which, though only being a small percentage of total activated fibre stiffness, could play a key role in maintaining the stability of the sarcomere structure at the start of contraction when only few crossbridges are formed. They relate the static tension to the 'residual force enhancement' that was observed in activated and stretched muscle fibres more than 60 years ago (Abbott and Aubert 1952), a subject that continues to be revisited on occasion, but has remained unresolved.

The article by Ger Stienen is a detailed and comprehensive review of the diverse ways in which the properties of the basic contractile unit in the heart can help preserve cardiac function or progressively lead to heart failure (Stienen 2014). The review starts with an overview of the different types of heart failure and the key facets of normal contractile function and regulation, including important quantitative consideration of the force, length and metabolic changes involved and the importance of passive tension, the latter being due primarily to titin, though in whole tissue it can also depend on collagen and fibrosis. Eight specific topics are considered, each starting with a description of normal function and then of pathological changes and dysfunction that can directly contribute to, or eventually otherwise lead to, heart failure, with distinction made between systolic and diastolic heart failure. These topics include the determinants of maximum tension and the rate of tension redevelopment, the calcium and length dependence of tension development, and the rate of ATP utilization. Importantly, the review is careful in setting out what is actually known, what is supposed, and what is not known. Overall, the review gives clear insights into the diverse mechanisms by which the precise contractile characteristics can lead to heart failure.

The final article by Canato and colleagues in the Reggiani group deals with the other key process determining muscle activation, namely the storage, release and reuptake of calcium ions by the sarcoplasmic reticulum (SR) (Canato et al. 2014). The review starts by giving an overview of the protein components and processes controlling SR calcium storage and release, and then describes specific human diseases arising from mutations in key proteins, in particular in the ryanodine receptor (Ryr1), the protein forming the actual calcium release channel in skeletal muscles. Mutations in Ryr1, and resulting aberrant Ryr1 function, can give rise to a diverse range of pathological conditions, including malignant hyperthermia (MH), a potentially fatal syndrome induced by anaesthetics in susceptible individuals, CCD, a congenital myopathy producing hypotonia and muscle weakness, and exertional or environmental heat-stroke (EHS), where heavy exercise and elevated environmental temperatures can trigger a hyperthermic crisis. The article then provides a comprehensive review of various mouse models of these diseases 
and the important mechanistic insights provided by these models. The review methodically sets out the quite diverse characteristics and likely underlying mechanisms in the different models, and also includes consideration of a quite different murine model in which calsequestrin (Casq1), the predominant calcium storage protein within the SR of skeletal muscles, is knocked out. In such mice exposure to anaesthetics or high environmental temperatures can also trigger crises resembling MH or EHS, leading the authors to speculate on how quite diverse and distinct molecular deficits can lead to a similar cascade of molecular events culminating in a muscle crisis.

We are very pleased to have had these interesting, high quality articles contributed to the EMC 2014 Special Issue. All the articles were rigorously reviewed by $2-3$ specialists, and revised accordingly. These articles attest to the considerable progress that has been made in our understanding of skeletal and cardiac muscle tissue and function, and in the processes underlying muscle diseases. We fully expect that this progress will continue, driven by the upand-coming generation of muscle researchers, and will be conveyed to the scientific community at the EMC and similar meetings, and in further Special Issue articles. Finally, we would like to thank all authors for their efforts and prompt writing and amending of their articles.

\section{References}

Abbott BC, Aubert XM (1952) The force exerted by active striated muscle during and after change of length. J Physiol 117:77-86
Beckendorf L, Linke WA (2014) Emerging importance of oxidative stress in regulating striatedmuscle elasticity. J Muscle Res Cell Motil.doi:10.1007/s10974-014-9392-y

Brücke E (1858) Untersuchungen über den Bau der Muskelfasern mit Hilfe des polarisierten Lichtes Denkschriften der kaiserlichen. Akademie der Wissenschaften Mathematisch-Naturwissenschaftliche Classe 15: 69-84

Canato M, Capitanio P, Reggiani C, Cancellara L (2014) The disorders of the calcium release unit of skeletal muscles: whathave we learned from mouse models? J Muscle Res Cell Motil.doi:10.1007/s10974-014-9396-7

Cecchi G, Griffiths PJ, Taylor S (1982) Muscular contraction: kinetics of crossbridge attachment studied by high-frequency stiffness measurements. Science 217:70-72

Ford LE, Huxley AF, Simmons RM (1981) The relation between stiffness and filament overlap in stimulated frog muscle fibres. J Physiol 311:219-249

Galler S (2014) Forgotten research from 19th century: science should not follow fashion. J Muscle Res Cell Motil. doi:10.1007/ s10974-014-9399-4

Krause W (1869) Die motorischen Endplatten der quergestreiften Muskelfasern. Hahn, Hannover

Rassier DE, Leite F, Nocella M, Cornachione A, Colombini B, Bagni MA (2014) Non-crossbridge forces in activated striated muscles: a titindependent mechanism of regulation? J Muscle Res Cell Motil. doi:10.1007/s10974-014-9397-6

Scellini B, Piroddi N, Flint GV, Regnier M, Poggesi C, Tesi C (2014) Impact of tropomyosin isoform composition on fast skeletalmuscle thin filament regulation and force development. J Muscle Res Cell Motil.doi:10.1007/s10974-014-9394-9

Stienen GJM (2014) Pathomechanisms in heart failure: the contractile connection. J Muscle Res Cell Motil.doi:10.1007/s10974-0149395-8 